(C) 2015 IEEE. Personal use of this material is permitted. Permission from IEEE must be obtained for all other uses, in any current or future media, including reprinting/republishing this material for advertising or promotional purposes, creating new collective works, for resale or redistribution to servers or lists, or reuse of any copyrighted component of this work in other works. 


\title{
Real-time Transmission of Panoramic Images for a Telepresence Wheelchair
}

\author{
Van Kha Ly Ha, Student Member, EMBS, IEEE, Tuan Nghia Nguyen, Member, IEEE, \\ and Hung T. Nguyen, Senior Member, IEEE
}

\begin{abstract}
This paper proposes an approach to transmit panoramic images in real-time for a telepresence wheelchair. The system can provide remote monitoring and assistive assistance for people with disabilities. This study exploits technological advancement in image processing, wireless communication networks, and healthcare systems. High resolution panoramic images are extracted from the camera which is mounted on the wheelchair. The panoramic images are streamed in real-time via a wireless network. The experimental results show that streaming speed is up to $250 \mathrm{KBps}$. The subjective quality assessments show that the received images are smooth during the streaming period. In addition, in terms of the objective image quality evaluation the average peak signalto-noise ratio of the reconstructed images is measured to be $39.19 \mathrm{~dB}$ which reveals high quality of images.
\end{abstract}

\section{INTRODUCTION}

In recent years, advancement in images processing and innovations in telecommunications have made significant impacts on human life. However, remote monitoring, social interaction, and healthcare support through a wheelchair are of practical interest but are the technical challenges [1].

Telepresence systems have recently become an emerging area research since they have been developed to overcome fixed video conference systems [2-4]. Recently, extensive research in the field of the telepresence has addressed the issues of the interaction between humans and robots in remote areas. The applications of telepresence robots have been focused on multimedia conferences and tele-education, see, e.g., [2], and references therein. Particularly, Giraff is a typical telepresence robot for remote visiting which has been developed for home care of the elderly [3]. Some early telepresence robots are already commercially available [4].

To the best of our knowledge, various telepresence robot schemes have been presented in the previous works [2-4], but no such investigation considers 360-degree view. The techniques developed for these systems typically use one camera or webcam to capture a single image. Remote users have to manipulate the view direction [2-4]. The majority of existing telepresence systems has not considered full view display or immersive visualisation. Therefore, it would be more effective if a 360-degree camera is built in the telepresence system. An immersive panorama TV service

Van Kha Ly Ha, Tuan Nghia Nguyen, Hung T. Nguyen are with the Centre for Health Technologies, Faculty of Engineering and Information Technology, University of Technology, Sydney. NSW 2007, Australia, (email: VanKha.L.Ha@student.uts.edu.au; tuannghia.nguyen@uts.edu.au; Hung.Nguyen@uts.edu.au). system was also introduced in [5]. This motives us to find an efficient method for the telepresence system using a 360degree camera.

In terms of smart wheelchairs, Nguyen, et al. developed an intelligent wheelchair which works as a hand-free wheelchair control system and provides obstacles detection while navigating through an unknown environment to assist wheelchair users [1]. However, a telepresence wheelchair has not been studied in the literature for far.

Despite the fact that telepresence robots and wheelchair systems exist, it is essential to develop a next generation wheelchair. The telepresence wheelchair is a mobile wheelchair platform which is capable of providing remote monitoring from an unknown starting location. This system is expected to be useful in remote monitoring and collaborative control to provide assistive support for people with disabilities. In this work, we develop and extend the work of [1] by adding a spherical vision camera to the wheelchair.

The main objective of this paper is to propose a unique system named a telepresence wheelchair. We investigate, design, and implement appropriate techniques to stream real world panoramic images surrounding a wheelchair from the spherical camera to a remote location via wireless networks in real-time. Particularly, we conduct real-time experiments in high resolution panoramic images extraction and partition the images into packets to be suitable for the physical layer of the wireless transmission channel and then apply the transmission control protocol/internet protocol (TCP/IP) for transmission. The streaming rate and image quality are also assessed by objective and subjective quality evaluations.

The remainder of this paper is organized as follows. In Section II, we introduce the proposed methodology in detail. Section III presents experiments and results of panoramic images processing and wireless streaming. Finally, conclusion and future works are drawn in Section IV.

\section{METHODOLOGY}

\section{A. Telepresence Wheelchair System}

A teleperesence wheelchair system is based on server and client topology as shown in Fig. 1. This system will process and transmit real world images around the wheelchair while it is moving to a remote location over wireless network in real-time. 


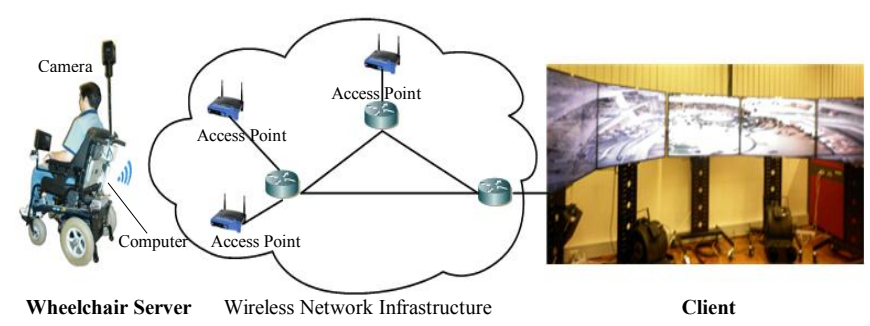

Figure 1. The telepresence wheelchair system topology.

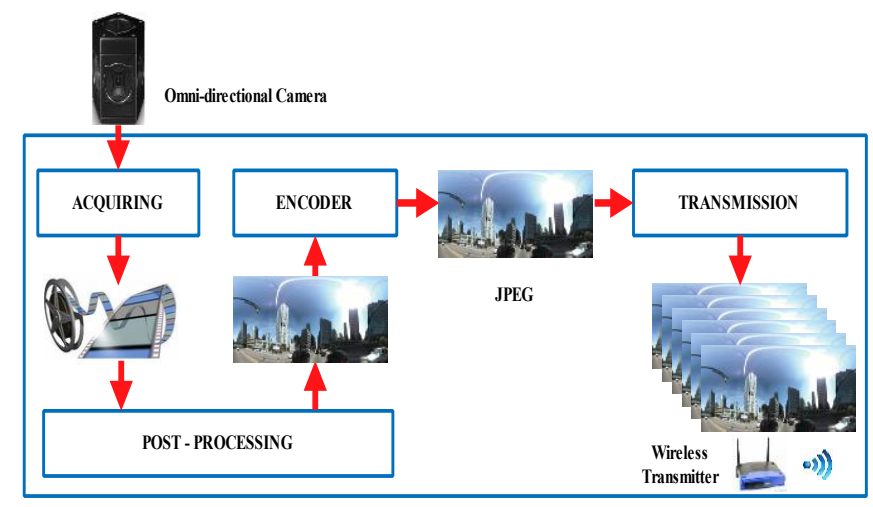

Figure 2. The operation of telepresence wheelchair server.

In this system, the server consists of a powered wheelchair which is equipped with: a Mac mini computer, control interfaces (joystick, head movement, thought control system), sensory systems (ultrasonic, laser URG-04LXbased) for obstacle detection and Ladybug3 camera. The Ladybug3 is an Omni-directional camera, designed and manufactured by Point Grey Research [6].

The operation of the telepresence wheelchair server is basically described by the diagram in Fig. 2. In this server, frames are acquired from Omni-directional camera, post processed and encoded. Due to the currently available technology and quality requirements, the original images demand compression methods to minimize the congestion of the network. Once the frame has been compressed and extracted it is ready to transmit over wireless networks.

\section{B. JPEG Image Coding}

To reduce the image data for efficient transmission, we exploit the image compression scheme, namely Joint Photographic Experts Group (JPEG). JPEG is an image compression method for image data transmission over the digital communication networks [7]. The key steps in JPEG encoding are outlined in Fig. 3. After image preparation, the uncompressed image samples are grouped into data units of $8 \times 8$ pixels and passed to color transform and downsampling to reduce the resolution of the chrominance channels. These data units of $8 \times 8$ shifted pixel values are defined by $S_{y x}$, where $x$ and $y$ are in the range of zero to seven. Each of these values is then transformed using Forward Discrete Cosine Transformation (FDCT). The FDCT is then applied to each transformed pixel value by (1). This transformation is carried out 64 times per data unit. The resulting 64 coefficients of $S_{v u}$ is given by (1).

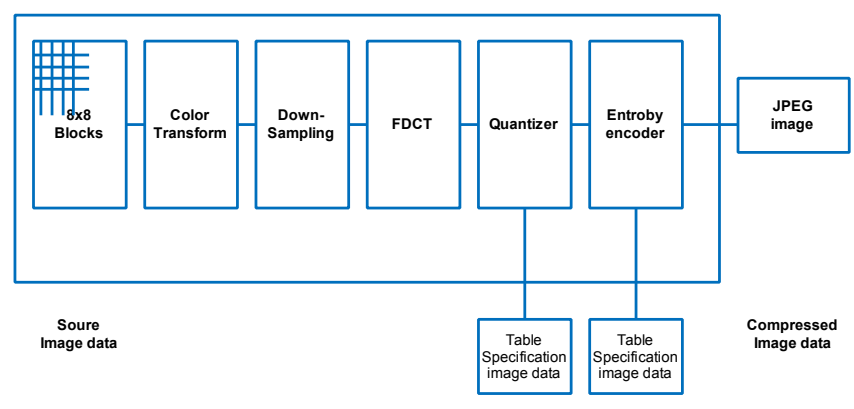

Figure 3. Block diagram of the JPEG Encoder.

$$
S_{v u}=\frac{1}{4} c_{u} c_{v} \sum_{x=0}^{7} \sum_{y=0}^{7} S_{y x} \cos \frac{(2 x+1) u \pi}{16} \cos \frac{(2 y+1) v \pi}{16} .
$$

For reconstruction of the image, the decoder uses the Inverse Discrete Cosine Transformation (IDCT). The coefficients $S_{v u}$ are used for the calculation:

$$
S_{y x}=\frac{1}{4} \sum_{v=0}^{7} \sum_{u=0}^{7} c_{u} c_{v} S_{v u} \cos \frac{(2 x+1) u \pi}{16} \cos \frac{(2 y+1) v \pi}{16}
$$

where $c_{u}, c_{v}=\frac{1}{\sqrt{2}}$ for $\mathrm{u}, \mathrm{v}=0$; otherwise $c_{u}, c_{v}=1$.

The quantization step is responsible for the source distortion in the codec and determines the compression rate. The performance measurement parameter, namely mean square error (MSE) is calculated by

$$
M S E=\frac{\sum_{i=0}^{M-1} \sum_{j=0}^{N-1}[I(\mathrm{i}, \mathrm{j})-K(\mathrm{i}, \mathrm{j})]^{2}}{M * N}
$$

where $M$ and $N$ represent the dimensions of the images. To measure the quality of reconstructed images, the peak signalto-noise ratio (PSNR) is calculated from the obtained MSE [8].

$$
\begin{aligned}
P S N R & =10 \log _{10}\left[\frac{M A X_{I}^{2}}{M S E}\right]=20 \log _{10}\left[\frac{M A X_{I}}{\sqrt{M S E}}\right] \\
& =20 \log _{10}\left(M A X_{I}\right)-10 \log _{10}(M S E)
\end{aligned}
$$

where $I(\mathrm{i}, \mathrm{j})$ is the original image, $K(\mathrm{i}, \mathrm{j})$ is reconstructed image. $M A X_{I}$ is 255 for an 8-bit image.

\section{Images Streaming Over Wireless Network}

Video streaming applications over wireless networks in real-time face various challenges, due to both the nature of the wireless radio channel and the stringent delivery requirements of media traffic. Bandwidth fluctuations in wireless channels result in not only packet losses, but also residual bit errors [8]. These losses and errors have severely adverse effect on the compressed image bit stream. In some cases, the decoder fails to recognize and recover the compressed image. Thus, it is highly desired that image streaming scheme has the ability to adapt the bit rate according to network conditions.

In this paper, TCP/IP is adopted for the transmission protocol because there is a guarantee of the reliable transmission. In order to minimize the distortion and overcome with errors on a compressed JPEG image streaming over wireless networks, there exist various error 
protection and error correction techniques. In our method, we handle the error problems by applying imagepacketization scheme, retransmission mechanism, and buffer control. The receiver checks the packet for any errors and sends a positive or a negative acknowledgment (ACK) to the transmitter depending on whether the packet is received correctly or erroneously. As the transmitter receives a negative ACK signal, it resends the packet. On the other hand if the transmitter receives a positive ACK signal, it sends the next packet. With such an error control, we can decrease packet losses and packet delivery to enhance the image quality. The main issue of this technique is that retransmission timeout may delay the streaming.

\section{EXPERIMENTS AND RESULTS}

Experiments were deployed at the University of Technology, Sydney. Matlab and Wireshark were used for analysing the performance and packet transmission. Software modules were developed and implemented by using C\#, C language in Visual Studio 2013 Integrated Development Environment (IDE).

\section{A. Images Acquisition and Processing}

The purpose of this work is to capture live images around the wheelchair from the camera in real time at a fast frame rate and high quality. Images are acquired via the IEEE 1394B fire wire cable to the Mac mini computer. If the compression is disabled, the camera transmits uncompressed images at transfer rates of $800 \mathrm{Mbit} / \mathrm{s}$ and at 30 frames per second (fps) to hard disk [6]. The output of this stage is a sequence of six images as shown in Fig. 4.

In our experiments, these six images were processed to be composited together to form a panoramic image. This obtained image was compressed into JPEG format in realtime for transmission. To evaluate the performance, we conducted the experiment to generate images with a resolution of $2048 \times 1024$ pixels into Bit map (BMP) and JPEG compression. The results confirm that our scheme provides significantly reduced capacity of JPEG (280 Kilobytes) in comparison with BMP (6,145 Kilobytes).

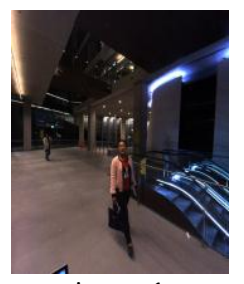

image 1

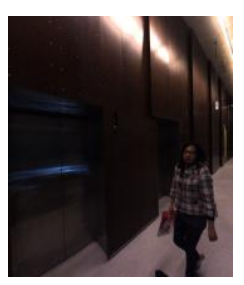

image 4

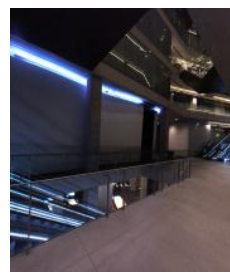

image 2

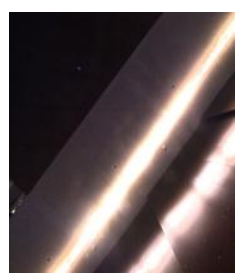

image 5

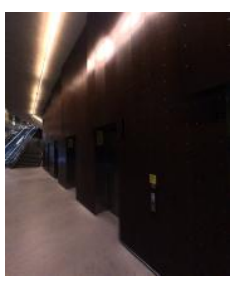

image 3

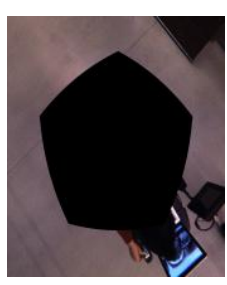

image 6
Figure 4. A sequence of six frames is extracted from six cameras.

\section{B. Real-time panoramic image streaming}

We carried out ten experiments at various locations to stream panoramic images with a resolution of $2048 \times 1024$ pixels from a wheelchair at zone 1 and zone 2 to a client at the remote location via a wireless network at the Centre for Health Technologies laboratory as shown in Fig. 5. The wheelchair battery is expected to last for 8 hours and should be charged overnight. The wheelchair speed was reduced to $5 \mathrm{~m} / \mathrm{s}$ to ensure the wireless connection could be maintained. In the experiments, we exploited the error detection, imagepacketization, retransmission, and buffer control scheme to overcome the packet loss and bit errors. Table I lists the parameters of system configuration.

TABLE I. NETWORK SETTINGS FOR EXPERIMENTS.

\begin{tabular}{|l|l|}
\hline \multicolumn{1}{|c|}{ Parameter } & \multicolumn{1}{c|}{ Value } \\
\hline \hline Wireless & IEEE $802.11 \mathrm{~b} / \mathrm{g} / \mathrm{n}$ in $2.4 \mathrm{GHz}$ \\
\hline Transport protocol & TCP/IP \\
\hline Bandwidth & $20 \mathrm{MHz}$ \\
\hline Number of Access Point & 2 \\
\hline Speed Requirement, Flow direction & Real-time, Duplex \\
\hline Vehicle speed & $5 \mathrm{~m} / \mathrm{s}$ \\
\hline
\end{tabular}

The experimental results demonstrate that the received images are of high quality as illustrated in Fig. 6 and above the quality PSNR threshold. The performance of image streaming is smooth at up to 250 Kilobytes per second (KBps) as shown in Fig. 7. It can be observed from Fig. 8 that the average PSNR of the whole sequence of one hundred frames at a distance of $45 \mathrm{~m}$ is $31.64 \mathrm{~dB}$.

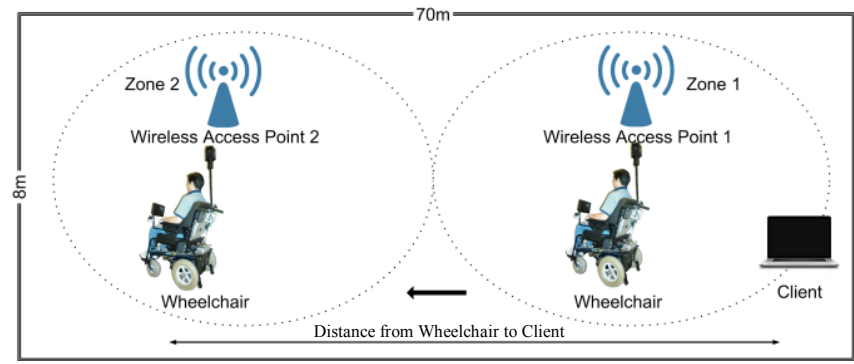

Figure 5. System performance test at the Centre for Health Technologies.



Figure 6. The image obtained over wireless with our scheme ${ }_{\text {Bytes/s }}$

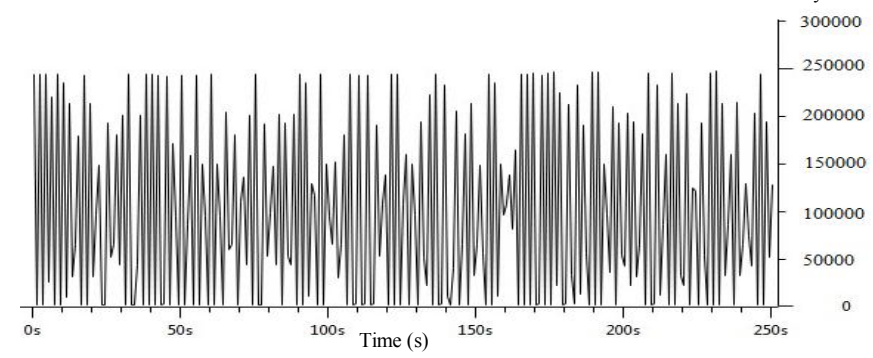

Figure 7. Streaming rates (Bytes/s) vs. Time (s). 




Figure 8. Received image quality was measured by PSNR.

TABLE II. AVERAGE PSNR RESULTS FOR VARIOUS DISTANCES.

\begin{tabular}{|c|c|c|c|c|}
\hline Trial Times & $\begin{array}{c}\text { Distance } \\
{[\mathbf{m}]}\end{array}$ & Access Point & $\begin{array}{c}\text { Average } \\
\text { MSE }\end{array}$ & $\begin{array}{c}\text { Average } \\
\text { PSNR [dB] }\end{array}$ \\
\hline \hline 1 & $5.00 \pm 1.00$ & 1 & 4.98 & 43.79 \\
\hline 2 & $10.00 \pm 1.00$ & 1 & 4.68 & 43.34 \\
\hline 3 & $15.00 \pm 1.00$ & 1 & 4.47 & 43.72 \\
\hline 4 & $20.00 \pm 1.00$ & 1 & 5.45 & 42.14 \\
\hline 5 & $25.00 \pm 1.00$ & 1 & 5.78 & 41.61 \\
\hline 6 & $30.00 \pm 1.00$ & 1 & 7.88 & 39.95 \\
\hline 7 & $35.00 \pm 1.00$ & 1 & 18.19 & 38.85 \\
\hline 8 & $40.00 \pm 1.00$ & 2 & 17.10 & 37.07 \\
\hline 9 & $45.00 \pm 1.00$ & 2 & 50.97 & 31.64 \\
\hline 10 & $50.00 \pm 1.00$ & 2 & 82.58 & 29.78 \\
\hline Mean & $27.50 \pm 1.00$ & 1.30 & 20.21 & 39.19 \\
\hline Minimum & $5.00 \pm 1.00$ & 1.00 & 4.47 & 29.78 \\
\hline Maximum & $50.00 \pm 1.00$ & 2.00 & 82.58 & 43.79 \\
\hline
\end{tabular}

Table II lists the results of wireless streaming with various distances from the client to the wheelchair which connected to the closest wireless access point (AP). It is clearly seen from Table II that the average PSNR is reduced when the wheelchair moves far away from the wireless access point. The average PSNR of the above ten experiments is 39.19 dB. In comparison with the previous study [9-12], it is worth noting that the quality of image is excellent for PSNR $\geq 37$ $\mathrm{dB}$ and is good for $32 \mathrm{~dB} \leq$ PSNR $<37 \mathrm{~dB}$. The results from Table II show that the proposed scheme obtains high quality images for the transmission distances of less than $35 \mathrm{~m}$ from one AP. The wheelchair is able to stream in a longer distance with two wireless access points in the same network. However, the wheelchair is mobile and the wireless link between nodes is rapidly changing over time. Due to the handover from $\mathrm{Wi}-\mathrm{Fi}$, the wheelchair has to disconnect with one AP and then reconnects with another stronger signal AP. As reselection wireless access point may result in reduced performance, we have implemented an algorithm for automatic network connection in order to overcome this challenge.

From the technical perspective, besides handling the huge amount of images extractions and compressions, the synchronization of buffer processing and data streaming is the major challenge. Nevertheless, the potential benefits of emerging 360-degree telepresence technologies and our positive outcomes will trigger the development of more advanced applications in human-wheelchair interaction, remote surveillance, telemedicine, and e-health systems.

\section{CONCLUSION}

We presented a novel telepresence wheelchair system. The state-of-the-art technology for high resolution panoramic image generation, compression and streaming over wireless networks in real-time was designed and implemented. The experimental results obtained on the wheelchair in a practical environment demonstrated the effectiveness of the images processing techniques. Streaming 360-degree panoramic images over wireless channels is the feasibility of designing and developing a telepresence wheelchair system. Future work will enhance the transmission speed and consider collaborative controls for remote interaction for practical applications.

\section{REFERENCES}

[1] J. S. Nguyen, S. W. Su, and H. T. Nguyen, "Experimental study on a smart wheelchair system using a combination of stereoscopic and spherical vision," in 35th Annual International Conference of the IEEE Engineering in Medicine and Biology Society (EMBC), 2013, pp. $4597-4600$

[2] K. Oh-Hun, K. Seong-Yong, K. Young-Geun, and K. Dong-Soo, "Telepresence robot system for English tutoring," in IEEE Workshop on Advanced Robotics and its Social Impacts (ARSO), 2010, pp. 152155.

[3] J. Gonzalez-Jimenez, C. Galindo, and J. R. Ruiz-Sarmiento, "Technical improvements of the Giraff telepresence robot based on users' evaluation," in IEEE Conference on RO-MAN, 2012, pp. 827-832.

[4] K. M. Tsui, A. Norton, D. J. Brooks, E. McCann, M. S. Medvedev, and H. A. Yanco, "Design and development of two generations of semiautonomous social telepresence robots," in IEEE International Conference on Technologies for Practical Robot Applications (TePRA), 2013, pp. 1-6.

[5] S. Dongmahn, K. Suhyun, Y. JaeWook, P. Hogun, and K. Heedong, "Immersive panorama TV service system," in IEEE International Conference on Consumer Electronics (ICCE), 2012, pp. 201-202.

[6] I. Point Grey Research. (2014, 2 June). Ladybug3 Available: http://www.ptgrey.com/products/ladybug3/ladybug3_360_video_camer a.asp

[7] ITU-T, "Recommendation T.81 Digital compression and coding of continuous-tone still images - Requirements and guidelines," ed: ITUT, 1993.

[8] M. G. Martini, R. S. H. Istepanian, M. Mazzotti, and N. Y. Philip, "Robust Multilayer Control for Enhanced Wireless Telemedical Video Streaming," IEEE Transactions on Mobile Computing, vol. 9, pp. 5$16,2010$.

[9] W. Xiaolin, S. Cheng, and X. Zixiang, "On packetization of embedded multimedia bitstreams," IEEE Transactions on Multimedia, vol. 3, pp. 132-140, 2001.

[10]I. Oleksii, "Using PSNR parameter as a measurement tool in real-time estimation of multimedia information in Wimax systems," in International Conference on Modern Problems of Radio Engineering Telecommunications and Computer Science (TCSET), 2012, pp. 283283.

[11]X. Yuwei, J. D. Deng, and M. Nowostawski, "Quality of service for video streaming over multi-hop wireless networks: Admission control approach based on analytical capacity estimation," in IEEE Eighth International Conference on Intelligent Sensors, Sensor Networks and Information Processing, 2013, pp. 345-350.

[12]Y. Xu, J. D. Deng, M. Nowostawski, and M. K. Purvis, "Optimized routing for video streaming in multi-hop wireless networks using analytical capacity estimation," Journal of Computer and System Sciences, vol. 81, pp. 145-157, 2015. 